\title{
Editorial for the special issue of knowledge discovery and management in engineering design and manufacturing
}

\author{
Ying Liu · Jenny Harding
}

Received: 18 November 2008 / Accepted: 19 November 2008 / Published online: 20 December 2008

(C) Springer Science+Business Media, LLC 2008

Knowledge discovery and management using various advanced techniques, e.g. data $\backslash$ text $\backslash$ web $\backslash$ multimediamining, computational neuroscience, ontology, and corporate search engine, in engineering design and manufacturing has emerged as a very new research area. Engineering design and manufacturing professionals are eager to enhance their information processing and knowledge management capabilities by adopting these advanced knowledge techniques. A series of challenging topics have emerged, including for example, the effective management of design knowledge in global contexts (e.g. outsourcing) at both personal and enterprise wide levels, knowledge integration from individual manufacturing processes into advanced systems through the application of data or text mining and ontology, and how to enhance the agility of manufacturing enterprises through the latest research in artificial intelligence, informatics, WWW and knowledge management. This Special Issue is therefore dedicated to innovative, state-of-the-art research, technology development and applications of knowledge discovery and management in the broad context of design and manufacturing.

The first paper, "Data Mining in Manufacturing: A Review Based on the Kind of Knowledge" by Choudhary, Harding and Tiwari, provides a comprehensive survey of the current state of data mining applications in manufacturing industry with a special emphasis on the kind of knowledge mined and the corresponding data mining functions dedicated to fulfill such discovery activities, including concept character-

\section{Y. Liu (凶)}

Department of Industrial and Systems Engineering, The Hong Kong Polytechnic University, Kowloon, Hong Kong SAR, China e-mail: mfyliu@polyu.edu.hk

\section{J. Harding}

Wolfson School of Mechanical \& Manufacturing Engineering, Loughborough University, Loughborough, Leicestershire, UK ization and description, association relation, classification, prediction and clustering. The paper stresses the increasing industrial awareness of the benefits of harnessing data mining as a powerful tool for extracting useful information and knowledge intelligently. Finally, the paper proposes a text mining approach to study the current focus of data mining applications in manufacturing industry, which provides a kind of validation for the observations mentioned in the survey. The authors have discovered that areas like supply chain management, production planning and control and integration of data mining within manufacturing system need further attention. This echos with our own concerns particularly on migrating data mining tools from individual manufacturing processes to a system perspective.

The remaining papers in this issue can be clustered into three groups. The first group focuses on the process improvement and optimization through data mining, in particular through swarm optimization and with the assistance of ontology. "Optimizing a Batch Manufacturing Process through Interpretable Data Mining Models" by Last, Danon, Biderman and Miron, presents a data mining based methodology for optimizing the yield of crystal growth, a complex batch manufacturing process. The authors have adopted predictive data mining techniques to induce a set of probabilistic rules from Information Network algorithms to indicate the likelihood of each defect as a function of interaction between the important control variables. Their study also reveals that an earlier application of this technique with an even smaller data set will result in a lower failure rate subsequently, largely due to its discovery of failure patterns and the corresponding optimization procedures that are undertaken to control the loss.

"A Particular Swarm Optimization Approach for Components Placement Inspection on Printed Circuit Boards" by Wu, Wang, Ip, Wang, Chan and Wang, reports an approach 
using an accelerated species based Particular Swarm Optimization (ASPSO) for multi-template matching in solving a multiple components PCB detection problem. The core of their method is to formulate the multiple component PCB detection into a multimodel optimization problem. Their experimental study reveals that using ASPSO, the computational time and searching rate of locating and matching captured images can be greatly reduced.

"A Product Design Ontology for Enhancing Shape Processing in Design Workflows" by Catalano, Camossi, Ferrandes, Cheutet and Sevilmis, describes their efforts in building and using a product design ontology to enhance information sharing in the shape design and processing workflow. The benefits of using an ontology, with the support of industrial domain metadata, to search and retrieve shaperelated information, and even to benchmark processing tools is highlighted in their implementation. The authors also stress the importance of having an automatic approach of semantic annotation in implementing the product ontology in order to reduce the tedious efforts required in manual approaches. We see that could be best complemented by a text mining approach.

The second group focuses more on using text mining and various advanced language processing techniques for knowledge discovery and management in design and manufacturing industry. "Advanced P-Tree Based K-Nearest Neighbors for Customer Preference Reasoning Analysis" by Li, Shi, Charastrakul and Zhou, tackles an important issue in the new product design and development process, i.e. identification of customer preference and motivation. The authors have proposed an advanced P-Tree based K-nearest Neighbor (AP$\mathrm{KNN}$ ) algorithm for text classification in order to capture the useful information from open-ended customer answers via an intelligent survey system. Their experimental study shows that the proposed AP-KNN is able to provide more salient and discriminative features in representing the customers' major concerns, which in the end significantly shorten the survey analysis time.

"Towards Automatic Causality Boundary Identification from Root Cause Analysis Reports" by Kim, Aurisicchio and Wallace, focuses on a challenging problem of automatically identifying the causality boundary by analyzing the engineering root cause reports. Coupled with a probability modeling of boundaries' distribution and the local context analysis of language convention occurred in the root cause reports, their approach is able to outperform the baseline system which is developed based on general language modeling in natural language processing. It is also expected that further improvements could be achieved if domain knowledge, the treasure of domain experts, could be integrated in all aspects of the local language clue analysis.

The last group of papers reports the current study of deploying knowledge sharing and management in manufac- turing industry. "A New Multi-agent System Framework for Tacit Knowledge Management in Manufacturing Supply Chains" by Al-Mutawah, Lee and Cheung, proposes that the tacit knowledge management issue in manufacturing supply chain management could be addressed using a multi-agent system. Their simulation results, which are generated based on Dempster-Shafer theory, indicate that the proposed framework outperforms conventional models using local GAs and it could be possible to protect the host organization from releasing sensitive tacit knowledge to competitors. Such an effort is perfectly aligned with the expectations outlined by Choudhary, Harding and Tiwari in the first paper of this Special Issue.

"Applying Semantic Web Technologies to Knowledge Sharing in Aerospace Engineering" by Dadzie, Bhagdev, Chakravarthy, Chapman, Iria, Lanfranchi, Magalhaes, Petrelli and Ciravegna, reports their latest efforts in applying Semantic Web technology to optimize the knowledge reuse and sharing in aerospace engineering. Centered at their approach, ontology is treated as the backbone for information modeling, integration, navigation, retrieval, sharing and reuse, where the information elements, e.g. concepts, metadata and properties, are captured either automatically from legacy document analysis or through a user-defined web interaction system. We, the Guest Editors, see such efforts, which take advantage of the latest research in machine learning, data, text and multimedia mining, information retrieval, semantic technology and knowledge management from individual to enterprise wide in design and manufacturing, will emerge as a major research and application paradigm in intelligent information processing and management at large. It is also worth mentioning that the system's deployment in a real industrial scenario merits applause.

The eight articles selected for this Special Issue were chosen after two rounds of reviews, from 16 intentions. In the first round, each paper was peer reviewed by multiple reviewers (minimally two), and in the second round the revised articles were reviewed by the Guest Editors. A third revision was requested for a small number of articles. Finally, we would like to take this opportunity to thank all authors for their excellent work contributing to this Special Issue, to the research community and industry. We are very grateful to the reviewers for offering their precious time and effort and for providing constructive comments. Particularly, we would like to express our sincere thanks to Prof. Andrew Kusiak, Editor-in-Chief, for giving us the opportunity to make this Special Issue a reality and for his advice and support throughout the whole year. Finally, we also thank Melissa J. Andersen from Springer for her editorial assistance. Without all of you, this Special Issue would not be possible. 